\section{Pattern of allergen sensitivity among bronchial asthma patients in Sohag governorate, Egypt}

\author{
Asmaa Nasr El-Din ${ }^{1}$ and Kamal Abdel Sattar Ata \\ ${ }^{1}$ Department of Microbiology \& Immunology, Faculty of \\ Medicine, Sohag, University, Egypt. \\ ${ }^{2}$ Department of Chest Diseases \& Tuberculosis, Faculty of \\ Medicine, Sohag, University, Egypt.
}

The Egyptian Journal of Immunology Volume 28 (4), 2021: 250-263. www.Ejimmunology.org

\begin{abstract}
The purpose of this study was to identify the pattern of allergen-sensitivity in asthmatic patient in Sohag governorate, Egypt, and to provide data for therapeutic management of those patients by immunotherapy. The study included patients with bronchial asthma diagnosed clinically and by pulmonary function tests. Patients were subjected skin prick test to diagnose their sensitization to different allergens including aero-allergens, contact, and food allergens. Clinical and demographic data of the patients were collected for correlating it with the pattern of the sensitivity. The most frequent aero-allergen reported in asthmatic patients was birch (38.7\%), followed by sunflower seeds and mixed grass (29.5\% and $26.4 \%$ respectively), While the most frequent contact allergen reported was common wasp venom followed by honey bee venom ( $25.7 \%$ and $20.6 \%$ respectively). the most frequently reported food allergen was banana followed by milk and Sollanaceae $(12.2 \%$, $11.5 \%$, and $11.0 \%$ respectively). In conclusion, our study provided useful information on the pattern of allergen sensitization in this part of the country; such information would be helpful in treating allergic patients.
\end{abstract}

Keywords: Asthma, Skin prick test, IgE-specific.

Date received: 23 September 2021; accepted: 28 September 2021

\section{Introduction}

Allergy (immunological hypersensitivity) includes different clinical forms and manifestations. ${ }^{1}$ Respiratory allergy is mediated by IgE and manifested as asthma and allergic rhinitis that are referred to as united airway diseases (UADs) because they show many common characteristics. ${ }^{2}$ The prevalence of allergy is increasing and affects $30-40 \%$ of population throughout the world. ${ }^{3}$ The WHO reported that about 300 million patients have asthma and 400 million patients have allergic rhinitis worldwide. ${ }^{4}$ In 2025 , it is predicted that 400 million of individuals will suffer from allergic asthma and 500 million from allergic rhinitis. $^{5,6}$

Allergic rhinitis and asthma are considered atopic disorders. Atopy is defined as a genetic predisposition to production of IgE upon exposure to an environmental allergen with subsequent development of allergic reaction. The first atopic manifestation appears in childhood as atopic dermatitis and can further progress to allergic rhinitis and/or asthma at school age; this transition is called the atopic 
march. ${ }^{7,8}$ The main criterion of atopy is the ongoing production of allergen-specific IgE. This phenomenon is called sensitization. Sensitization is indispensible for diagnosis allergic diseases. The natural history of allergy is characterized by an increasing number and amplitude of sensitizations which is called polysensitization phenomenon. ${ }^{9}$

Asthma is defined as a heterogeneous, chronic inflammatory disease of the respiratory tract that leads to transient variable symptoms, such as wheezing, shortness of breath, chest tightness, coughing in alternating intensity, and expiratory airflow limitation defined by lowered forced expiratory volume. ${ }^{10}$ Asthma causes long-term morbidity, ${ }^{11}$ restrictions in the patient's quality of life, and high costs of hospitalization, medication, and work/school absenteeism. ${ }^{12}$

Detection of IgE-specific antibody against the offending allergens can be conducted in vivo by skin prick test (SPT) or in vitro by IgE-specific serum measurement. ${ }^{13}$ World Allergy Organization (WAO) stated that skin prick test is the gold standard in detecting IgE-specific antibodies. ${ }^{14}$ SPT is considered the first line diagnostic method, and specific-lgE assays as second line, SPT is cheap, quick, and sensitive. However, it has some drawbacks as it may cause severe local skin reaction and anaphylactic risk. ${ }^{15}$

The types of inhalant allergens that predominate in the environment of the patient differ widely depending on locality and can also vary within the same country. ${ }^{16}$ So, it is important to determine the local allergy pattern in patients of allergy and asthma. This study aimed at identifying the pattern of allergen sensitivity among patients with bronchial asthma using SPT against various indoor and outdoor inhalant and allergens. The study emphasis was on the impact of gender, age, and demographic data on the pattern of allergens responsible.

\section{Patients and Methods}

This cross-sectional study was conducted during the period from March 2019 to March 2021. It included 417 patients diagnosed to have bronchial asthma and visited the outpatient clinic of chest diseases and tuberculosis department of Sohag University hospitals. Age, gender, residence, occupation, socioeconomic status, and marital status data were recorded.

Inclusion criteria: Patients were included in the study if they were diagnosed with asthma; defined as a clinical syndrome consisting of wheeze, breathlessness, chest tightness and sometimes cough and confirmed by pulmonary function tests. Their Age ranged between 14 and 59 years.

Exclusion criteria included the following: Children, less than 14 years of age were not included as they might develop automatic remission beyond this age. Patients who had taken short-acting oral antihistaminic agents, beta-blockers, steroids, tricyclic antidepressants or any other drug that could affect the test within one week prior to testing were excluded. Patients on long-acting oral antihistaminic agents before four weeks of testing. Patients receiving corticosteroids 7 days before performing the SPT. Pregnant and lactating woman were excluded from this study. Finally, patients with history of severe life-threatening exacerbations of asthma.

All the study patients underwent SPT by using standard allergens obtained from Stallergenes (Paris, France). A total of 34 antigens were used which included three types of pollens; Birch, mixed grasses, and sun flower seeds. Three types of mites; Dermatophagoids pteronyssinus, Dermatophagoids farinae, house dust mites (HDM). Five types of fungi; Alternaria alternate, Aspergilus niger, Aspergilllus flavus, Aspergilllus fumigatus and penicillium notatum. Three types of insects; Honeybee venom, common wasp venom, and cockroach. Five types of pet dander and wool; sheep wool, dog dander and cat dander. Fifteen types of food items; fish, chicken, milk, egg white, maize, wheat, nuts, garlic, tomato, Sollanaceae, banana, chocolate. Histamine was used as a positive control, and buffered saline was used as a negative control to rule out dermographism, which makes the test results difficult to interpret. 
The skin prick test: The selected allergens were dropped on a marked area on the volar side of the forearm, and the area was pricked using Stallerpoint (Stallergenes, Paris, France) at least $2 \mathrm{~cm}$ apart. After 15 minutes, the area was wiped. Results were evaluated by measuring wheal and flare reaction for each allergen. A positive reaction was defined as the presence of a wheal and flare. Wheals twofold larger in diameter than the histamine control or showing pseudopods were classified as $4+$. If the wheal was $3 \mathrm{~mm}$ or up to two times larger than the histamine control, it was classified as $3+$. More than one-half the size of the positive control it was scored as 2+. More than one-quarter of the size of the positive control it was scored as 1+; and less than one-quarter of the size of the positive control scored as 0 . Scores of $2+$ or more were defined as positive reactions ${ }^{17}$.

\section{Statistical analysis}

Data was analyzed using STATA version 14.2 (Stata Statistical Software: Release 14.2 College Station, TX: StataCorp LP.). Data were presented as number and percentage and compared using either Chi square test or fisher exact test. The strength of association of different allergen with each other was measured using cross tabulation and Kendall rank correlation test. Graphs were produced by using the Excel program. $\mathrm{P}$ value was considered significant if it was less than 0.05 . The correlation coefficient takes on values ranging between +1 and -1 . The following points were the accepted guidelines for interpreting the correlation coefficient: Values between 0 and 0.3 ( 0 and -0.3 ) indicated a weak positive (negative) linear relationship. Values between 0.3 and 0.7 (0.3 and -0.7) indicated a moderate positive (negative) linear relationship. Values between 0.7 and $1.0(-0.7$ and -1.0$)$ indicated a strong positive (negative) linear relationship.

\section{Results}

The study included a total of 417 asthmatic patients; 182 females (43.6\%) and 235 males (56.4\%). The age of patients ranged from 14 to 65 years, The age categories of the patients included in the study were as follows; 172 (41.2\%) patients were (14-<25 years of age), $173(41.4 \%)$ patients ( $25-<45$ years of age), and 71 (17\%) patients $(45-<65$ years of age). Residence and occupations of the patients were recorded. Patients live in rural environment were 190 (45.9\%), while patients residing in urban environment accounted for 226 (54.1\%).

The most frequent aero-allergen reported in asthmatic patients was birch (38.7\%), followed by sunflower seeds and mixed grass $(29.5 \%$ and $26.4 \%$ respectively), While the most frequent contact allergen reported was common wasp venom followed by honey bee venom ( $25.7 \%$ and $20.6 \%$ respectively). The most frequently reported food allergen was banana followed by milk and Sollanaceae (12.2\%, 11.5\%, and $11.0 \%$, respectively). (Tables 1, 2, and 3) (Figures 1, 2).

The frequency of sensitivity to aeroallergens in various age groups was as determined: the most frequently reported sensitivity in all age groups was to birch, while the lowest percentages were to Aspergillus niger, Penicillium notatum, Aspergillus fumigatus. Sensitivity to common wasp venom was more frequently observed in the age group $(45-\leq 65)$. However, sensitivity to honeybee venom and cockroaches was more frequent in the age group ( $25-\leq 45$ years of age). The sensitivity to milk and egg white was higher in the age group (14- $\leq 25$ years of age). The sensitivity to banana and Sollanaceae was higher in the age group ( $25-\leq 45$ years of age). There was no significant difference in the frequency of allergy to the same allergen in different age groups except for Dermatophagoids farina $(P=0.002)$. (Table 4). 
Table 1. Frequency of sensitivity to aeroallergens in asthmatic patients

\begin{tabular}{lc}
\hline Allergen & Number (\%) \\
\hline Birch & $161(38.70 \%)$ \\
\hline Sunflower seeds & $123(29.57 \%)$ \\
\hline Mixed gases & $110(26.44 \%)$ \\
\hline House dust mites (HDM) & $80(19.23 \%)$ \\
\hline Alternaria alternate & $64(15.38 \%)$ \\
\hline Dermatophagoids pteronyssinus & $63(15.14 \%)$ \\
\hline Aspergillus fumigatus & $58(13.94 \%)$ \\
\hline Dermatophagoides farina & $46(11.06 \%)$ \\
\hline Aspergillus flavus & $19(4.57 \%)$ \\
\hline Penicillium notatum & $14(3.37 \%)$ \\
\hline Aspergillus niger & $8(1.92 \%)$ \\
\hline
\end{tabular}

Table 2. Frequency of sensitivity to contact in asthmatic patients.

\begin{tabular}{lc}
\hline Allergen & Number (\%) \\
\hline Common wasp venom & $107(25.72 \%)$ \\
\hline Honeybee venom & $86(20.67 \%)$ \\
\hline Cockroaches & $73(17.55 \%)$ \\
\hline Sheep wool & $7(1.68 \%)$ \\
\hline Dog dander & $7(1.68 \%)$ \\
\hline Cat dander & $5(1.20 \%)$ \\
\hline
\end{tabular}

Table 3. Frequency of sensitivity to food in asthmatic patients

\begin{tabular}{lc}
\hline Allergen & Number (\%) \\
\hline Banana & $51(12.26 \%)$ \\
\hline Milk & $48(11.54 \%)$ \\
\hline Sollanaceae & $46(11.06 \%)$ \\
\hline Tomato white & $35(8.41 \%)$ \\
\hline Fish & $28(6.73 \%)$ \\
\hline Nuts & $20(4.81 \%)$ \\
\hline Garlic & $16(3.85 \%)$ \\
\hline Wheat flour & $10(2.40 \%)$ \\
\hline Chicken & $8(1.92 \%)$ \\
\hline Chocolate & $8(1.92 \%)$ \\
\hline Maize & $8(1.92 \%)$ \\
\hline
\end{tabular}




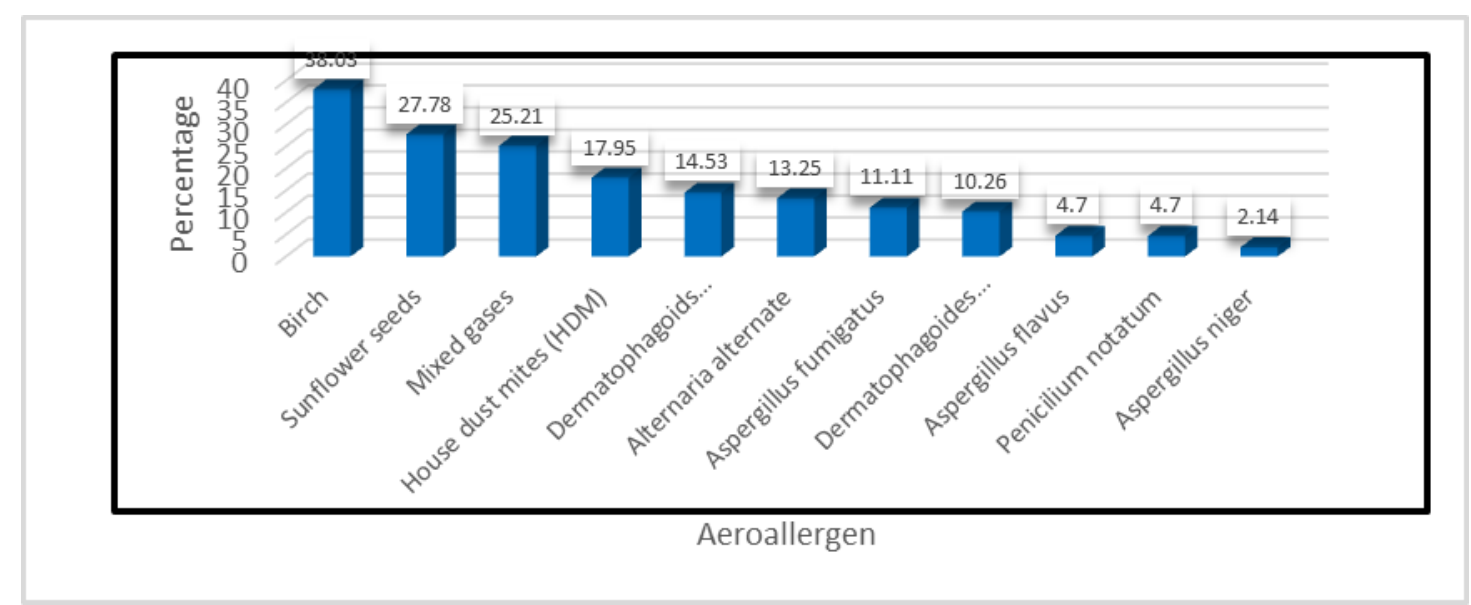

a)

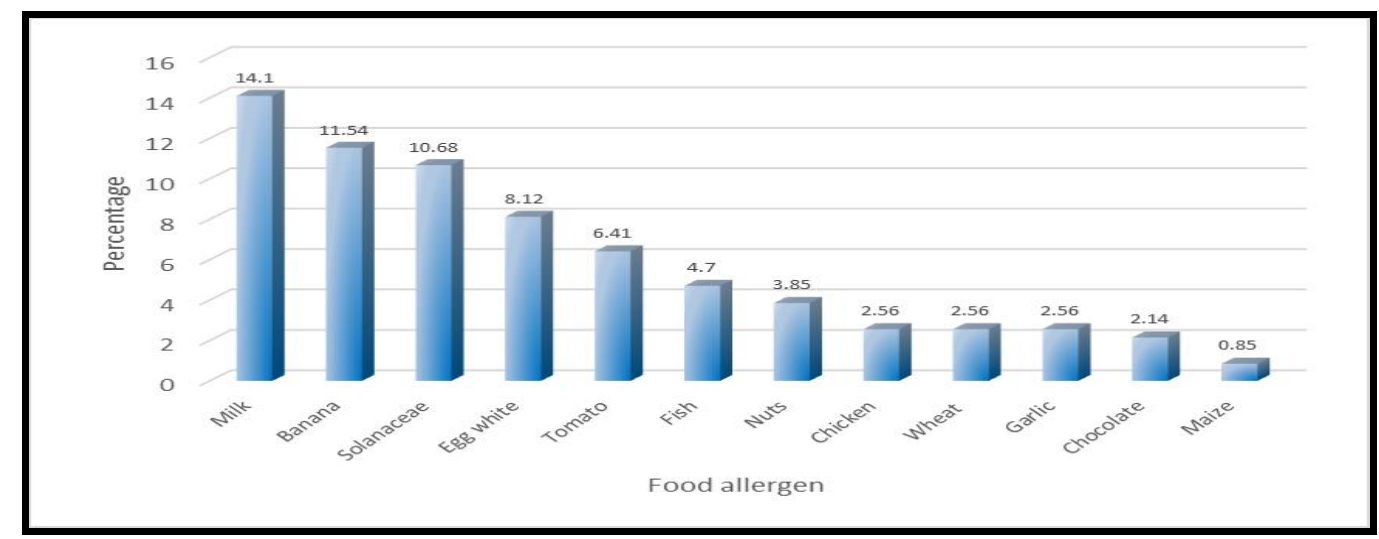

b)

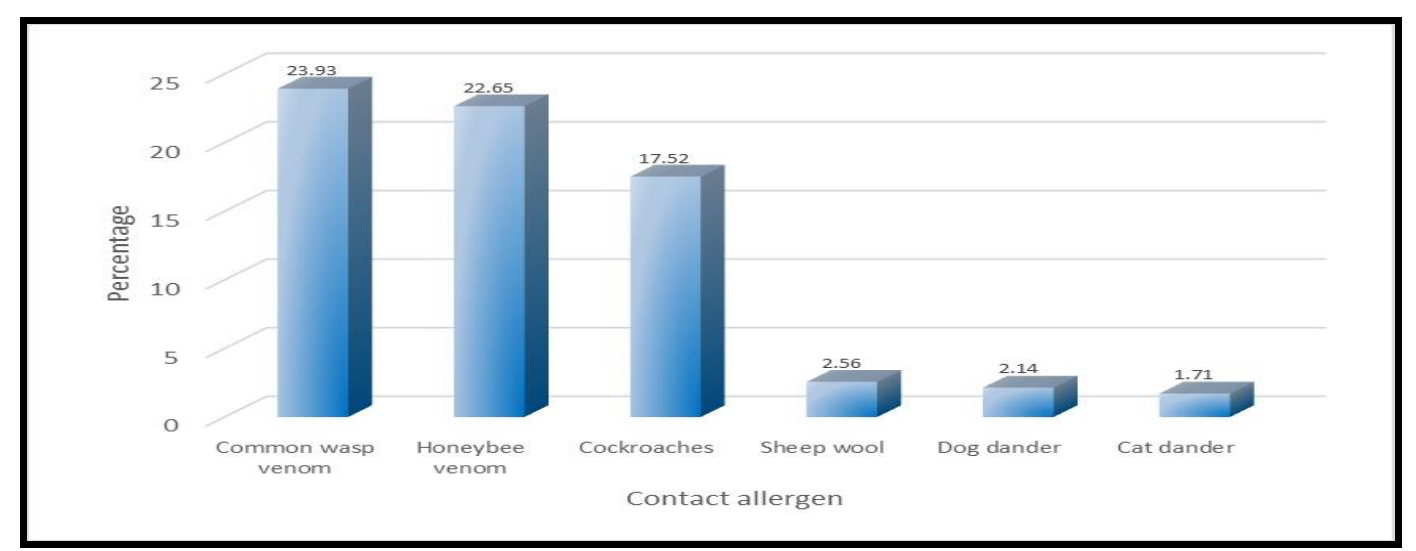

c)

Figure 1. Distribution of sensitivity to various allergens in male asthmatic patients: a) Aeroallergens, b) Food allergens, and c) Contact allergens 


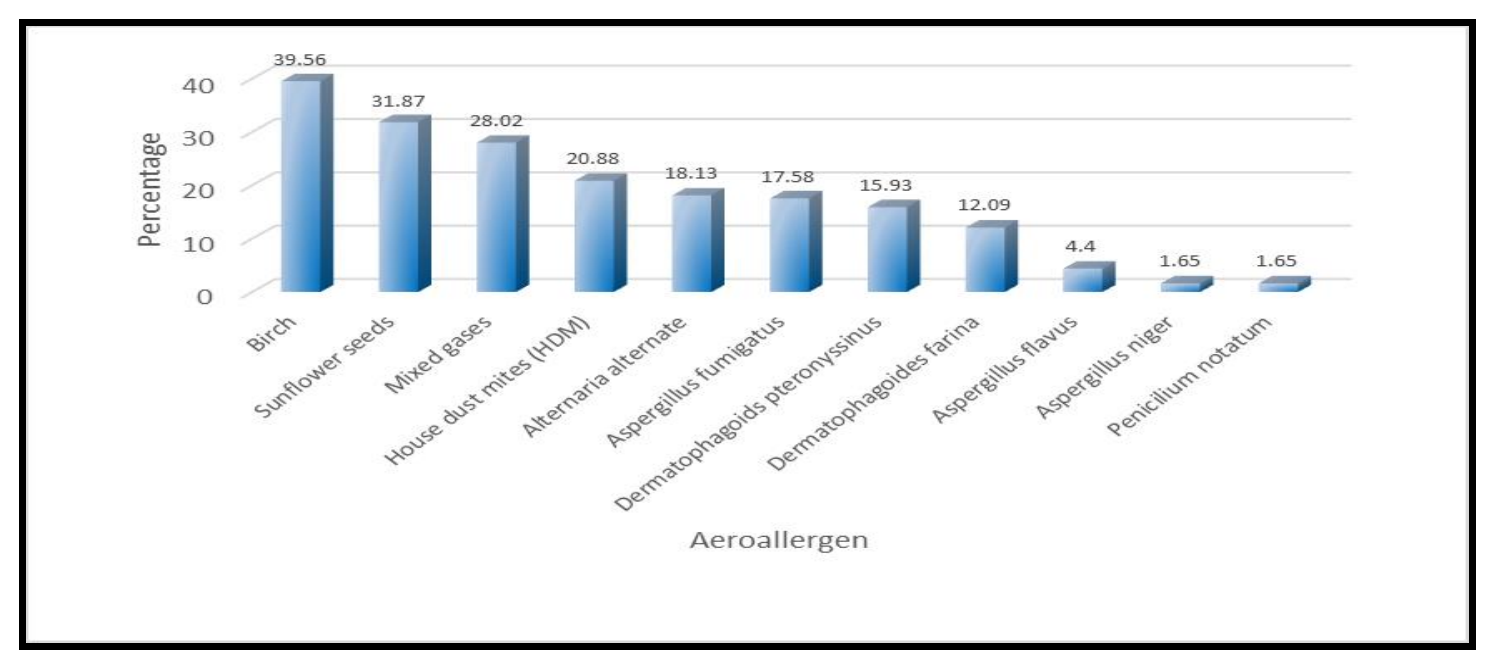

a)

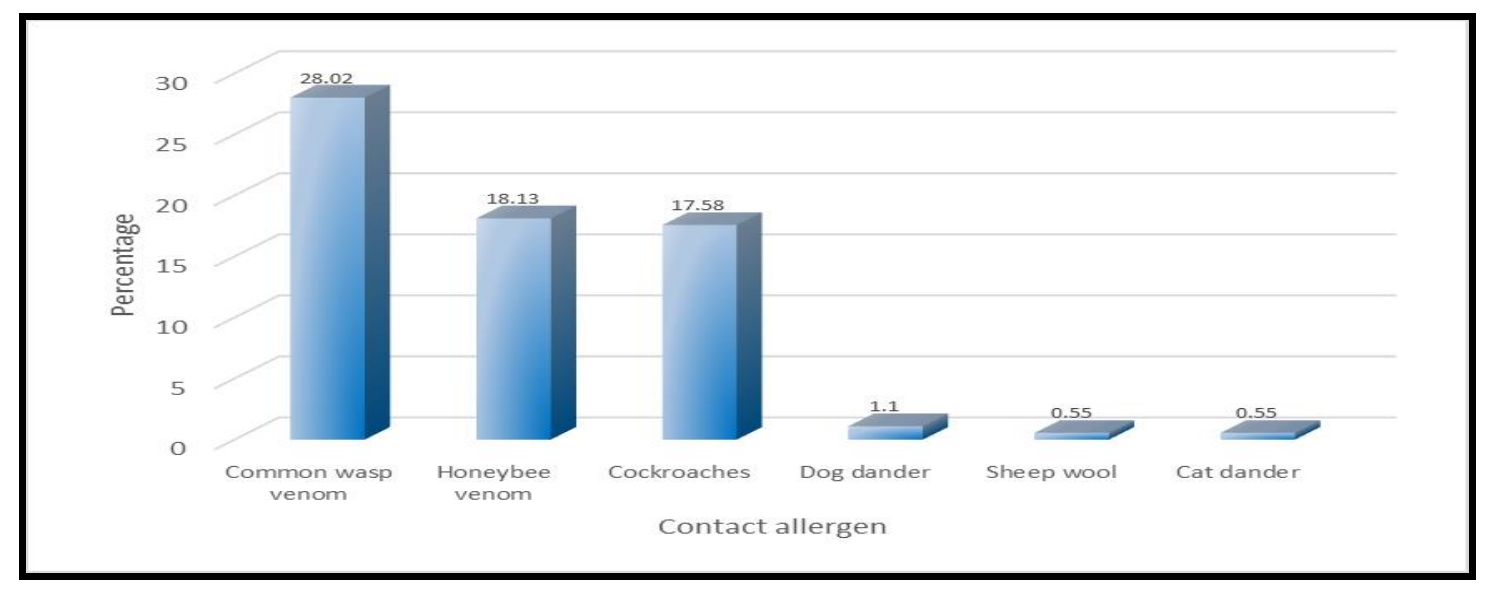

b)

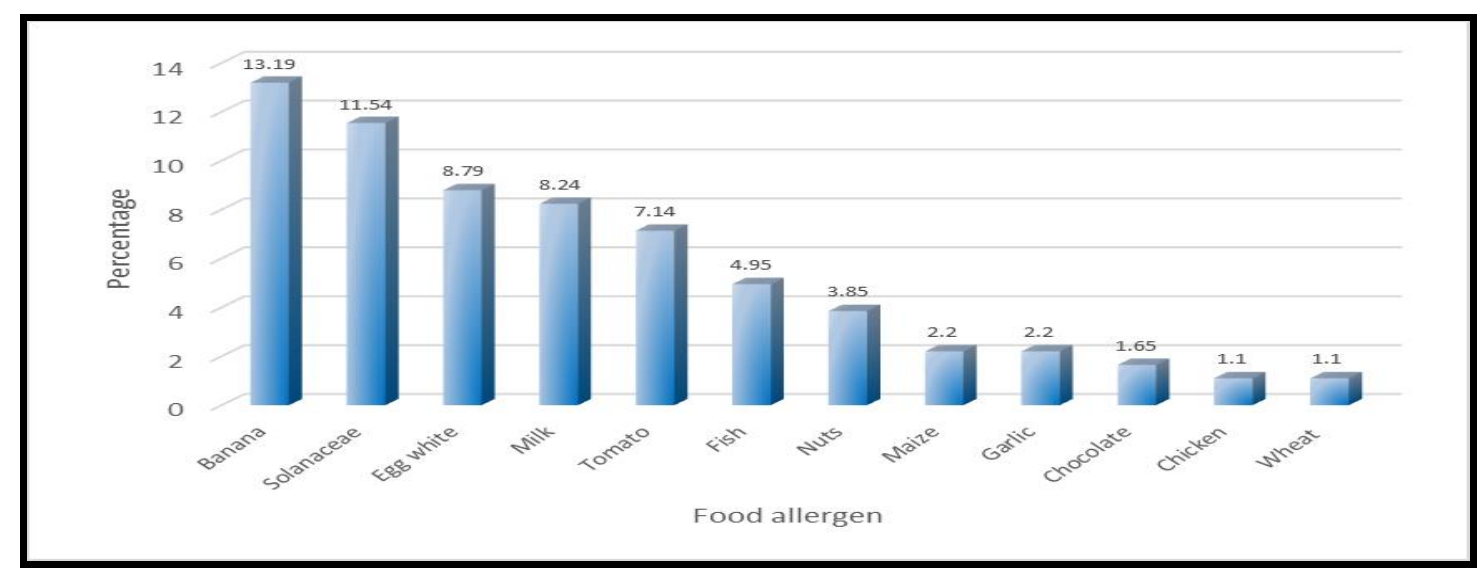

c)

Figure 2. Distribution of sensitivity to various allergens in female asthmatic patients: a) Aeroallergens, b) contact allergens, and c) food allergens 
Table 4. Relation between age and frequency of sensitivity to different allergens

\begin{tabular}{|c|c|c|c|c|}
\hline Allergen & $\begin{array}{c}\text { Age }(12-<25 \\
\text { years) } \\
\mathrm{N}=172\end{array}$ & $\begin{array}{c}\text { Age }(25-<45 \\
\text { years) } \\
\mathrm{N}=173\end{array}$ & $\begin{array}{c}\text { Age }(45-\leq 65 \\
\text { years) } \\
N=71\end{array}$ & $P$ value \\
\hline \multicolumn{5}{|l|}{ Aeroallergen } \\
\hline Dermatophagoides farina & $8(4.65 \%)$ & 27 (15.61\%) & 11 (15.49\%) & 0.002 \\
\hline Dermatophagoids pteronyssinus & $20(11.63 \%)$ & $35(20.23 \%)$ & $8(11.27 \%)$ & 0.051 \\
\hline House dust mites (HDM) & $31(18.02 \%)$ & $36(20.81 \%)$ & $13(18.31 \%)$ & 0.79 \\
\hline Aspergillus flavus & $10(5.81 \%)$ & $7(4.05 \%)$ & $2(2.82 \%)$ & 0.54 \\
\hline Aspergillus niger & $5(2.91 \%)$ & $3(1.73 \%)$ & 0 & 0.32 \\
\hline Aspergillus fumigatus & $23(13.37 \%)$ & $26(15.03 \%)$ & $9(12.68 \%)$ & 0.86 \\
\hline Alternaria alternate & $26(15.12 \%)$ & $31(17.92 \%)$ & $7(0.28 \%)$ & 0.28 \\
\hline Penicillium notatum & $7(4.07 \%)$ & $5(2.89 \%)$ & $2(2.82 \%)$ & 0.80 \\
\hline Birch & $59(34.30 \%)$ & $75(43.35 \%)$ & $27(38.03 \%)$ & 0.22 \\
\hline Sunflower seeds & $45(26.16 \%)$ & $52(30.06 \%)$ & $26(36.62 \%)$ & 0.26 \\
\hline Mixed gas & $42(24.42 \%)$ & $52(30.06 \%)$ & $16(22.54 \%)$ & 0.35 \\
\hline \multicolumn{5}{|l|}{ Contact allergen } \\
\hline Honeybee venom & 30 (17.44\%) & 40 (23.12\%) & 16 (22.54\%) & 0.39 \\
\hline Common wasp venom & $34(19.77 \%)$ & $51(29.48 \%)$ & $22(30.99 \%)$ & 0.06 \\
\hline Cockroaches & $22(12.79 \%)$ & $36(20.81 \%)$ & $15(21.13 \%)$ & 0.10 \\
\hline Sheep wool & $5(2.91 \%)$ & $1(0.58 \%)$ & $1(1.41 \%)$ & 0.24 \\
\hline Cat dander & $3(1.74 \%)$ & $2(1.16 \%)$ & 0 & 0.52 \\
\hline Dog dander & $2(1.16 \%)$ & $4(2.31 \%)$ & $1(1.41 \%)$ & 0.70 \\
\hline \multicolumn{5}{|l|}{ Food allergen } \\
\hline Fish & 9 (5.23\%) & $6(3.47 \%)$ & 5 (7.04\%) & 0.47 \\
\hline Chicken & $3(1.74 \%)$ & $2(1.16 \%)$ & $3(4.23 \%)$ & 0.28 \\
\hline Egg white & $18(10.47 \%)$ & $13(7.51 \%)$ & $4(5.63 \%)$ & 0.40 \\
\hline Milk & 20 (11.63\%) & $16(9.25 \%)$ & $12(16.90 \%)$ & 0.24 \\
\hline Maize & $2(1.16 \%)$ & $3(1.73 \%)$ & $1(1.41 \%)$ & 0.91 \\
\hline Wheat flour & $2(1.16 \%)$ & $4(2.31 \%)$ & $2(2.82 \%)$ & 0.62 \\
\hline Nuts & $9(5.23 \%)$ & $3(1.73 \%)$ & $4(5.63 \%)$ & 0.17 \\
\hline Chocolate & $3(1.74 \%)$ & $3(1.73 \%)$ & $2(2.82 \%)$ & 0.83 \\
\hline Banana & $19(11.05 \%)$ & $21(12.14 \%)$ & $11(15.49 \%)$ & 0.63 \\
\hline Sollanaceae & $17(9.88 \%)$ & $21(12.14 \%)$ & $8(11.27 \%)$ & 0.80 \\
\hline Tomato & $13(7.56 \%)$ & $13(7.51 \%)$ & $2(2.82 \%)$ & 0.35 \\
\hline Garlic & $4(2.33 \%)$ & $4(2.31 \%)$ & $2(2.82 \%)$ & 0.97 \\
\hline
\end{tabular}

$P<0.05$ is significant.

The sensitivity to mites, birch, sun flower seeds, mixed grass, and most of contact and food allergens was higher in male asthmatic patients than in females but the difference did not reach a statistical significance (Table 5). Furthermore, no significant difference was observed in the frequency of sensitivity to various allergens in relation to urban versus rural environments, except for allergy to Dermatophagoids farina, Aspergillus niger, and Alternaria alternata which were more frequently reported in employees who live in an urban environment. (Table 6). 
Table 5. Relation between sex and frequency of sensitivity to different allergens.

\begin{tabular}{|c|c|c|c|}
\hline Allergen & $\begin{array}{l}\text { Female } \\
\mathrm{N}=182\end{array}$ & $\begin{array}{c}\text { Male } \\
\mathrm{N}=234\end{array}$ & $P$ value \\
\hline \multicolumn{4}{|l|}{ Aeroallergen } \\
\hline Dermatophagoides farina & $22(12.09 \%)$ & $24(10.26 \%)$ & 0.56 \\
\hline Dermatophagoids pteronyssinus & 29 (15.93\%) & $34(14.53 \%)$ & 0.69 \\
\hline House dust mites (HDM) & $38(20.88 \%)$ & $42(17.95 \%)$ & 0.45 \\
\hline Aspergillus flavus & $8(4.40 \%)$ & $11(4.70 \%)$ & 0.88 \\
\hline Aspergillus niger & $3(1.65 \%)$ & $5(2.14 \%)$ & 1.00 \\
\hline Aspergillus fumigatus & $32(17.58 \%)$ & $26(11.11 \%)$ & 0.06 \\
\hline Alternaria alternate & $33(18.13 \%)$ & $31(13.25 \%)$ & 0.17 \\
\hline Penicillium notatum & $3(1.65 \%)$ & $11(4.70 \%)$ & 0.09 \\
\hline Birch & $72(39.56 \%)$ & $89(38.03 \%)$ & 0.75 \\
\hline Sunflower seeds & $58(31.87 \%)$ & $65(27.78 \%)$ & 0.36 \\
\hline Mixed gas & $51(28.02 \%)$ & $59(25.21 \%)$ & 0.52 \\
\hline \multicolumn{4}{|l|}{ Contact allergen } \\
\hline Honeybee venom & $33(18.13 \%)$ & $53(22.65 \%)$ & 0.26 \\
\hline Common wasp venom & $51(28.02 \%)$ & $56(23.93 \%)$ & 0.34 \\
\hline Cockroaches & $32(17.58 \%)$ & $41(17.52 \%)$ & 0.99 \\
\hline Sheep wool & $1(0.55 \%)$ & $6(2.56 \%)$ & 0.14 \\
\hline Cat dander & $1(0.55 \%)$ & $4(1.71 \%)$ & 0.39 \\
\hline Dog dander & $2(1.10 \%)$ & $5(2.14 \%)$ & 0.48 \\
\hline \multicolumn{4}{|l|}{ Food allergen } \\
\hline Fish & $9(4.95 \%)$ & $11(4.70 \%)$ & 0.91 \\
\hline Chicken & $2(1.10 \%)$ & $6(2.56 \%)$ & 0.48 \\
\hline Egg white & $16(8.79 \%)$ & $19(8.12 \%)$ & 0.81 \\
\hline Milk & 15 (8.24\%) & $33(14.10 \%)$ & 0.06 \\
\hline Maize & $4(2.20 \%)$ & $2(0.85 \%)$ & 0.41 \\
\hline Wheat flour & $2(1.10 \%)$ & $6(2.56 \%)$ & 0.48 \\
\hline Nuts & $7(3.85 \%)$ & 9 (3.85\%) & 1.00 \\
\hline Chocolate & $3(1.65 \%)$ & $5(2.14 \%)$ & 0.72 \\
\hline Banana & 24 (13.19\%) & 27 (11.54\%) & 0.61 \\
\hline Sollanaceae & 21 (11.54\%) & 25 (10.68\%) & 0.78 \\
\hline Tomato & $13(7.14 \%)$ & 15 (6.41\%) & 0.77 \\
\hline Garlic & $4(2.20 \%)$ & $6(2.56 \%)$ & 0.81 \\
\hline
\end{tabular}

$P<0.05$ is significant. 
Table 6. Relation between residence and frequency of sensitivity to different allergens.

\begin{tabular}{|c|c|c|c|}
\hline Allergen & $\begin{array}{c}\text { Rural } \\
\mathrm{N}=190\end{array}$ & $\begin{array}{l}\text { Urban } \\
\mathrm{N}=226\end{array}$ & $P$ value \\
\hline \multicolumn{4}{|l|}{ Aeroallergen } \\
\hline Dermatophagoides farina & $19(10.00 \%)$ & 27 (11.95\%) & 0.53 \\
\hline Dermatophagoids pteronyssinus & $27(14.21 \%)$ & 36 (15.93\%) & 0.63 \\
\hline House dust mites (HDM) & 36 (18.95\%) & $44(19.47 \%)$ & 0.89 \\
\hline Aspergillus flavus & $6(3.16 \%)$ & $13(5.75 \%)$ & 0.21 \\
\hline Aspergillus niger & $5(2.63 \%)$ & $3(1.33 \%)$ & 0.48 \\
\hline Aspergillus fumigatus & $23(12.11 \%)$ & $35(15.49 \%)$ & 0.32 \\
\hline Alternaria alternate & $32(16.84 \%)$ & $32(14.16 \%)$ & 0.45 \\
\hline Penicillium notatum & $7(3.68 \%)$ & $7(3.10 \%)$ & 0.74 \\
\hline Birch & $76(40.00 \%)$ & $85(37.61 \%)$ & 0.62 \\
\hline Sunflower seeds & $62(32.63 \%)$ & $61(26.99 \%)$ & 0.21 \\
\hline Mixed gases & $50(26.32 \%)$ & $60(26.55 \%)$ & 0.96 \\
\hline \multicolumn{4}{|l|}{ Contact allergen } \\
\hline Honeybee venom & $36(18.95 \%)$ & $50(22.12 \%)$ & 0.43 \\
\hline Common wasp venom & $48(25.26 \%)$ & $59(26.11 \%)$ & 0.85 \\
\hline Cockroaches & $34(17.89 \%)$ & $39(17.26 \%)$ & 0.87 \\
\hline Sheep wool & $5(2.63 \%)$ & $2(0.88 \%)$ & 0.25 \\
\hline Cat dander & $5(2.63 \%)$ & 0 & 0.02 \\
\hline Dog dander & $4(2.11 \%)$ & $3(1.33 \%)$ & 0.71 \\
\hline \multicolumn{4}{|l|}{ Food allergen } \\
\hline Fish & $14(7.37 \%)$ & $6(2.65 \%)$ & 0.03 \\
\hline Chicken & $4(2.11 \%)$ & $4(1.77 \%)$ & 1.00 \\
\hline Egg white & 15 (7.89\%) & 20 (8.85\%) & 0.73 \\
\hline Milk & $19(10.00 \%)$ & $29(12.83 \%)$ & 0.37 \\
\hline Maize & $1(0.53 \%)$ & $5(2.21 \%)$ & 0.23 \\
\hline Wheat flour & $4(2.11 \%)$ & $4(1.77 \%)$ & 1.00 \\
\hline Nuts & $9(4.74 \%)$ & $7(3.10 \%)$ & 0.39 \\
\hline Chocolate & 4 (2.11\%) & 4 (1.77\%) & 1.00 \\
\hline Banana & $26(13.68 \%)$ & 25 (11.06\%) & 0.42 \\
\hline Sollanaceae & $23(12.11 \%)$ & $23(10.18 \%)$ & 0.53 \\
\hline Tomato & $13(6.84 \%)$ & 15 (6.64\%) & 0.93 \\
\hline Garlic & $5(2.63 \%)$ & $5(2.12 \%)$ & 1.00 \\
\hline
\end{tabular}

$P<0.05$ is significant.

Assessment of the association between sensitivity to various aeroallergens: There was a statistically significant positive correlation between the sensitivity to Dermatophagoids farina and Dermatophagoids pteronyssinus, which indicated that common allergy to both of them is frequently detected $(P<0.0001)$.
In addition, there was a significant positive correlation between Aspergillus niger and Alternaria alternata $(P<0.0001)$. The highest significant positive association was between allergy to birch, sunflower seeds, and mixed grass $(P<0.0001)$. As regards to contact allergens there was a statistically significant positive correlation between allergy to common 
wasp venom and honeybee venom, and between allergy to common wasp venom and cockroaches ( $P 0.001$ and 0.0001 , respectively. In food allergens it was noticed that there was moderate positive correlation between milk and egg white allergy ( $r=0.46, P<0.0001)$, and weak positive correlation between allergy to tomato and Sollanaceae $(r=0.33, P<0.0001)$. (Tables 7, 8 , and 9).

Table 7. Association between allergies to various aeroallergens.

\begin{tabular}{|c|c|c|c|c|c|c|c|c|c|c|}
\hline Allergen & $\begin{array}{l}\text { Dermato- } \\
\text { phagoides } \\
\text { farina }\end{array}$ & $\begin{array}{l}\text { Dermato- } \\
\text { phagoids } \\
\text { pteronyssinus }\end{array}$ & $\begin{array}{l}\text { House } \\
\text { dust } \\
\text { mites } \\
\text { (HDM) }\end{array}$ & $\begin{array}{l}\text { Aspergillus } \\
\text { flavus }\end{array}$ & $\begin{array}{l}\text { Aspergillus } \\
\text { niger }\end{array}$ & $\begin{array}{l}\text { Aspergillus } \\
\text { fumigatus }\end{array}$ & $\begin{array}{l}\text { Alternaria } \\
\text { alternate }\end{array}$ & $\begin{array}{l}\text { Penicillium } \\
\text { notatum }\end{array}$ & Birch & $\begin{array}{l}\text { Sunflower } \\
\text { seeds }\end{array}$ \\
\hline $\begin{array}{l}\text { Dermatophagoids } \\
\text { pteronyssinus }\end{array}$ & $\begin{array}{l}r=0.54 \\
P<0.001\end{array}$ & & & & & & & & & \\
\hline $\begin{array}{l}\text { House dust mites } \\
\text { (HDM) }\end{array}$ & $\begin{array}{l}\mathrm{r}=0.02 \\
P=0.65\end{array}$ & $\begin{array}{l}\mathrm{r}=-0.05 \\
P=0.28\end{array}$ & & & & & & & & \\
\hline Aspergillus flavus & $\begin{array}{l}\mathrm{r}=0.03 \\
P=0.50\end{array}$ & $\begin{array}{l}\mathrm{r}=0.10 \\
P=0.04\end{array}$ & $\begin{array}{l}\mathrm{r}=-0.02 \\
P=0.70\end{array}$ & & & & & & & \\
\hline Aspergillus niger & $\begin{array}{l}\mathrm{r}=-0.05 \\
P=0.32\end{array}$ & $\begin{array}{l}\mathrm{r}=-0.05 \\
P=0.23\end{array}$ & $\begin{array}{l}r=-0.09 \\
p=0.16\end{array}$ & $\begin{array}{l}r=-0.03 \\
P=0.54\end{array}$ & & & & & & \\
\hline $\begin{array}{l}\text { Aspergillus } \\
\text { fumigatus }\end{array}$ & $\begin{array}{l}\mathrm{r}=-0.03 \\
P=0.52\end{array}$ & $\begin{array}{l}\mathrm{r}=-0.09 \\
P=0.06\end{array}$ & $\begin{array}{l}\mathrm{r}=-0.04 \\
P=0.44\end{array}$ & $\begin{array}{l}\text { R0.01= } \\
P=0.81\end{array}$ & $\begin{array}{l}\mathrm{r}=0.04 \\
P=0.36\end{array}$ & & & & & \\
\hline $\begin{array}{l}\text { Alternaria } \\
\text { alternate }\end{array}$ & $\begin{array}{l}\mathrm{r}=-0.02 \\
P=0.64\end{array}$ & $\begin{array}{l}\mathrm{r}=0.02 \\
P=0.62\end{array}$ & $\begin{array}{l}\mathrm{r}=-0.02 \\
P=0.65\end{array}$ & $\begin{array}{l}\mathrm{r}=-0.03 \\
P=0.55\end{array}$ & $\begin{array}{l}\mathrm{r}=0.33 \\
P<0.0001\end{array}$ & $\begin{array}{l}\mathrm{r}=0.002 \\
P=0.98\end{array}$ & & & & \\
\hline $\begin{array}{l}\text { Penicillium } \\
\text { notatum }\end{array}$ & $\begin{array}{l}\mathrm{r}=-0.03 \\
P=0.64\end{array}$ & $\begin{array}{l}\mathrm{r}=0.04 \\
P=0.40\end{array}$ & $\begin{array}{l}\mathrm{r}=0.02 \\
P=0.63\end{array}$ & $\begin{array}{l}\mathrm{r}=-0.04 \\
P=0.41\end{array}$ & $\begin{array}{l}\mathrm{r}=-0.03 \\
P=0.60\end{array}$ & $\begin{array}{l}\mathrm{r}=-0.04 \\
P=0.46\end{array}$ & $\begin{array}{l}\mathrm{r}=0.07 \\
P=0.17\end{array}$ & & & \\
\hline Birch & $\begin{array}{l}\mathrm{r}=-0.03 \\
P=0.56\end{array}$ & $\begin{array}{l}\mathrm{r}=0.10 \\
P=0.03\end{array}$ & $\begin{array}{l}\mathrm{r}=-0.01 \\
P=0.81\end{array}$ & $\begin{array}{l}\mathrm{r}=-1.10 \\
P=0.04\end{array}$ & $\begin{array}{l}\mathrm{r}=-0.004 \\
P=0.95\end{array}$ & $\begin{array}{l}\mathrm{r}=-0.09 \\
P=0.06\end{array}$ & $\begin{array}{l}\mathrm{r}=-0.07 \\
P=0.18\end{array}$ & $\begin{array}{l}\mathrm{r}=0.10 \\
P=0.046\end{array}$ & & \\
\hline Sunflower seeds & $\begin{array}{l}\mathrm{r}=-0.01 \\
P=0.84\end{array}$ & $\begin{array}{l}r=-0.04 \\
P=0.43\end{array}$ & $\begin{array}{l}\mathrm{r}=-0.05 \\
P=0.32\end{array}$ & $\begin{array}{l}\mathrm{r}=-0.02 \\
P=-0.75\end{array}$ & $\begin{array}{l}\mathrm{r}=-0.01 \\
P=0.78\end{array}$ & $\begin{array}{l}\mathrm{r}=-0.02 \\
P=0.72\end{array}$ & $\begin{array}{l}\mathrm{r}=-0.03 \\
P=0.57\end{array}$ & $\begin{array}{l}r=0.05 \\
P=0.27\end{array}$ & $\begin{array}{l}\mathrm{r}=0.31 \\
P<0.001\end{array}$ & \\
\hline Mixed gases & $\begin{array}{l}\mathrm{r}=0.03 \\
P=0.52\end{array}$ & $\begin{array}{l}\mathrm{r}=0.02 \\
P=0.68\end{array}$ & $\begin{array}{l}\mathrm{r}=-0.02 \\
P=0.75\end{array}$ & $\begin{array}{l}\mathrm{r}=-0.08 \\
P=0.11\end{array}$ & $\begin{array}{l}\mathrm{r}=0.07 \\
P=0.13\end{array}$ & $\begin{array}{l}\mathrm{r}=-0.10 \\
P=0.04\end{array}$ & $\begin{array}{l}\mathrm{r}=0.03 \\
P=0.52\end{array}$ & $\begin{array}{l}\mathrm{r}=0.04 \\
P=0.43\end{array}$ & $\begin{array}{l}\mathrm{r}=0.58 \\
P<0.0001\end{array}$ & $\begin{array}{l}\mathrm{r}=0.20 \\
P<0.0001\end{array}$ \\
\hline
\end{tabular}

Table 8. Association of different contact allergen with each other.

\begin{tabular}{|c|c|c|c|c|c|}
\hline Allergen & $\begin{array}{c}\text { Honeybee } \\
\text { venom }\end{array}$ & $\begin{array}{c}\text { Common wasp } \\
\text { venom }\end{array}$ & Cockroaches & Sheep wool & Cat dander \\
\hline \multicolumn{6}{|l|}{$\begin{array}{l}\text { Honeybee } \\
\text { venom }\end{array}$} \\
\hline Common & $r=0.03$ & & & & \\
\hline wasp venom & $P=0.001$ & & & & \\
\hline \multirow{2}{*}{ Cockroaches } & $r=-0.03$ & $r=0.35$ & & & \\
\hline & $P=0.51$ & $P=0.01$ & & & \\
\hline \multirow{2}{*}{ Sheep wool } & $r=0.03$ & $r=0.009$ & $r=-0.01$ & & \\
\hline & $P=0.60$ & $P=0.86$ & $P=0.82$ & & \\
\hline \multirow{2}{*}{ Cat dander } & $r=0.05$ & $r=-0.06$ & $r=0.007$ & $r=0.16$ & \\
\hline & $P=0.28$ & $P=0.19$ & $P=0.89$ & $P=0.001$ & \\
\hline \multirow{2}{*}{ Dog dander } & $r=-0.02$ & $r=0.05$ & $r=-0.01$ & $r=-0.02$ & $r=-0.01$ \\
\hline & $P=0.68$ & $P=0.30$ & $P=0.82$ & $P=0.73$ & $P=0.78$ \\
\hline
\end{tabular}

$P<0.05$ is significant. 
Table 9. Association of different food allergen with each other.

\begin{tabular}{|c|c|c|c|c|c|c|c|c|c|c|c|}
\hline Allergen & Fish & Chicken & Egg white & Milk & Maize & Wheat flour & Nuts & Chocolate & Banana & Sollanoceae & Tomato \\
\hline Chicken & $\begin{array}{l}r=0.05 \\
P=0.31\end{array}$ & & & & & & & & & & \\
\hline Milk & $\begin{array}{l}r=-0.05 \\
P=0.35\end{array}$ & $\begin{array}{l}r=-0.05 \\
P=0.30\end{array}$ & $\begin{array}{l}r=0.46 \\
P<0.0001\end{array}$ & & & & & & & & \\
\hline Maize & $\begin{array}{l}r=-0.03 \\
P=0.58\end{array}$ & $\begin{array}{l}r=-0.02 \\
P=0.74\end{array}$ & $\begin{array}{l}r=-0.04 \\
P=0.46\end{array}$ & $\begin{array}{l}\mathrm{r}=0.02 \\
P=0.69\end{array}$ & & & & & & & \\
\hline Wheat flour & $\begin{array}{l}r=-0.03 \\
P=0.52\end{array}$ & $\begin{array}{l}\mathrm{r}=-0.02 \\
P=0.69\end{array}$ & $\begin{array}{l}\mathrm{r}=0.02 \\
P=0.68\end{array}$ & $\begin{array}{l}r=0.004 \\
P=0.93\end{array}$ & $\begin{array}{l}r= \\
P=\end{array}$ & & & & & & \\
\hline Nuts & $\begin{array}{l}r=0.07 \\
P=0.14\end{array}$ & $\begin{array}{l}r=-0.03 \\
P=0.57\end{array}$ & $\begin{array}{l}r=-0.02 \\
P=0.75\end{array}$ & $\begin{array}{l}r=0.006 \\
P=0.90\end{array}$ & $\begin{array}{l}r=-0.02 \\
P=0.63\end{array}$ & $\begin{array}{l}r=0.06 \\
P=0.20\end{array}$ & & & & & \\
\hline Chocolate & $\begin{array}{l}r=-0.03 \\
P=0.52\end{array}$ & $\begin{array}{l}\mathrm{r}=-0.02 \\
P=0.69\end{array}$ & $\begin{array}{l}\mathrm{r}=-0.04 \\
P=0.39\end{array}$ & $\begin{array}{l}r=-0.05 \\
P=0.30\end{array}$ & $\begin{array}{l}r=0.02 \\
P=0.74\end{array}$ & $\begin{array}{l}r=-0.02 \\
P=0.69\end{array}$ & $\begin{array}{l}\mathrm{r}=0.06 \\
P=0.20\end{array}$ & & & & \\
\hline Banana & $\begin{array}{l}r=0.02 \\
P=0.70\end{array}$ & $\begin{array}{l}r=0.001 \\
P=0.99\end{array}$ & $\begin{array}{l}r=-0.09 \\
P=0.08\end{array}$ & $\begin{array}{l}r=-0.09 \\
P=0.07\end{array}$ & $\begin{array}{l}r=0.08 \\
P=0.11\end{array}$ & $\begin{array}{l}\mathrm{r}=0.11 \\
P=0.03\end{array}$ & $\begin{array}{l}r=-0.04 \\
P=0.46\end{array}$ & $\begin{array}{l}r=0.05 \\
P=0.027\end{array}$ & & & \\
\hline Solloneceas & $\begin{array}{l}r=-0.01 \\
P=0.88\end{array}$ & $\begin{array}{l}r=-0.05 \\
P=0.32\end{array}$ & $\begin{array}{l}r=-0.08 \\
P=0.11\end{array}$ & $\begin{array}{l}r=-0.03 \\
P=0.57\end{array}$ & $\begin{array}{l}r=0.09 \\
P=0.08\end{array}$ & $\begin{array}{l}r=0.06 \\
P=0.21\end{array}$ & $\begin{array}{l}r=-0.07 \\
P=0.15\end{array}$ & $\begin{array}{l}r=0.06 \\
P=0.21\end{array}$ & $\begin{array}{l}\mathrm{r}=0.50 \\
P<0.0001\end{array}$ & & \\
\hline Tomato & $\begin{array}{l}r=-0.02 \\
P=0.75\end{array}$ & $\begin{array}{l}r=-0.04 \\
P=0.45\end{array}$ & $\begin{array}{l}r=-0.08 \\
P=0.10\end{array}$ & $\begin{array}{l}r=-0.007 \\
P=0.89\end{array}$ & $\begin{array}{l}r=-0.03 \\
P=0.51\end{array}$ & $\begin{array}{l}r=0.03 \\
P=0.51\end{array}$ & $\begin{array}{l}r=-0.004 \\
P=0.94\end{array}$ & $\begin{array}{l}r=-0.04 \\
P=0.45\end{array}$ & $\begin{array}{l}r=0.51 \\
P<0.0001\end{array}$ & $\begin{array}{l}r=0.33 \\
P<0.0001\end{array}$ & \\
\hline Garlic & $\begin{array}{l}r=-0.04 \\
P=0.47\end{array}$ & $\begin{array}{l}r=-0.02 \\
P=0.66\end{array}$ & $\begin{array}{l}r=0.009 \\
P=0.86\end{array}$ & $\begin{array}{l}r=0.04 \\
P=0.40\end{array}$ & $\begin{array}{l}r=0.02 \\
P=0.74\end{array}$ & $\begin{array}{l}r=0.06 \\
P=0.21\end{array}$ & $\begin{array}{l}r=0.05 \\
P=0.31\end{array}$ & $\begin{array}{l}r=-0.02 \\
P=0.66\end{array}$ & $\begin{array}{l}r=0.08 \\
P=0.08\end{array}$ & $\begin{array}{l}r=0.04 \\
P=0.36\end{array}$ & $\begin{array}{l}r=0.02 \\
P=0.68\end{array}$ \\
\hline
\end{tabular}

$P<0.05$ is significant

\section{Discussion}

Atopy is a risk factor for bronchial responsiveness and exposure to the offending allergens can be precipitating factor in initiation and exaggeration of the attacks and even for sudden respiratory arrest. ${ }^{18,}{ }^{19}$ Hence, identification of the specific responsible allergens that are most prevalent in the locality of the patient is valuable for diagnosis and treatment of bronchial asthma. SPT is a commonly used method for detecting the implicated allergens and provides faster results compared to Radio Allegro Sorbent Testing (RAST). ${ }^{20}$

In the present study, the most frequent aero-allergen observed in asthmatic patients was birch (38.7\%), followed by sunflower seeds and mixed grass (29.5\% and $26.4 \%$ respectively). Furthermore, the most frequent contact allergen reported was common wasp venom followed by honey bee venom $(25.7 \%$ and 20.6\% respectively). However, a study conducted by Bharti Chogtu et al., $2017^{21}$ reported different findings as: the most common allergen was insects followed by dust, grass and tree pollens, fungus. Many studies reported that insects are the most common offending antigens, with percentage ranging from $17.5 \%$ to $43.9 \%$ of the overall antigens that have been studied. ${ }^{22-24}$ Two studies conducted in India, reported similar data to our study findings, The first study was done in Allahabad, Uttar Pradesh, on a relatively smaller cohort (50 patients), ${ }^{21}$ and the second study conducted in a medical college in Lucknow by Prasad et al., $2009,{ }^{23}$ reported data in agreement with our study results. While the first study revealed that dust mite $(78 \%)$ was the most common offending allergen followed by dust (66\%) and insects (44\%), the second study reported that the pollen of Amaranthus spinosus was the most common allergen (39.5\%). Note that the difference between data of various studies could be due to variations in study sample size and the geographical regions where they were conducted.

In our study $17.5 \%$ of male- and femaleasthmatic patients reported allergy to cockroaches, such findings were in accordance with several other studies. Agrawal et al., 2008, 
from Uttar Pradesh, India stated that cockroach was the most common offending insect in asthmatic patients, ${ }^{25}$ Sarinho et al., 2009, in a study from Brazil, conducted on adolescents, identified cockroach as the most common allergen. ${ }^{27}$ Also, a study conducted in Malaysia on 200 asthmatic patients revealed that cockroach was the most common allergen demonstrating skin positivity. Cockroachderived allergens come from its saliva and fecal material. ${ }^{19}$ In contrast to our results, however, a study conducted by Bharti Chogtu et al., 2017, ${ }^{21}$ reported that the commonest skin positivity against insects was to rice weevil as well as to grasshopper, followed by housefly and cockroach. They explained that their findings were due to the presence of protease allergens in the extracts obtained from reported insects, considered the cause of allergy. ${ }^{15-17}$ Also, in contrast to our study findings, a study done by Kumar $\mathrm{R}$ et al., $2012^{24}$ reported that the most common insect allergen was moth followed by mosquito. ${ }^{24}$ Patel et al., 2012, has also reported that moth was the most common insect allergen. $^{26}$

Allergy to fungi in our study was due to Alternaria alternate in $18.1 \%$ of female asthmatic patients and in $13.2 \%$ of male patients, While $17.5 \%$ of females and $11.1 \%$ of males were sensitive to Aspergilus fumigatus. The predominant fungus in a study of Bharti Chogtu et al., $2017^{21}$ were Aspergillus flavus followed by Aspergillus fumigates and Candida. Also, some other studies have shown that Aspergillus fumigatus was the most common fungus. ${ }^{23,25}$ A Study conducted on 180 poultry workers by Sarinho et al., $2009^{27}$ showed the predominance of sensitivity to Aspergillus niger.

Asthma and food allergy are frequently coexisting and both are increasing in prevalence. $^{28}$ In a Dutch cross sectional study with asthmatic children, half of the parents reported an allergic reaction to different types of food in their child's history. ${ }^{28}$ In our study, the most common food allergy detected was due to banana (13.1\% of females and $11.5 \%$ of males), followed by milk ( $8.2 \%$ of females and $14.1 \%$ of males) and Solaneceae $(11.5 \%$ of females and $10.6 \%$ of males). In a study conducted by Liu et al.,2010 29 stated that the most common food allergies in adults were shellfish $(2 \%)$, scaled fish $(0.4 \%)$, peanut $(0.6 \%)$, and tree nuts $(0.5 \%)$.

IgE directed to cross-reactive allergens may be responsible for both respiratory reactions and allergic reactions to food. Cross-reactivity between pollen and allergens from fruits and vegetables is the best-known example of the link between inhalant and food allergy. ${ }^{29}$ This information can explain the frequent presence of allergy to pollens in conjunction with allergy to banana and Solaneceae which was the commonest food allergy in our study.

In our study, we have measured the strength of association of different allergen with each other and we noticed the presence of a statistically significant positive association between allergy to Dermatophagoids farina and Dermatophagoids pteronyssinus ( $r=0.54$, $P<0.001)$. This could be explained by the common presence of both types of mites in the patient environment. There was also a statistically significant positive correlation between allergy to birch and sun flower seeds ( $r=0.31, P<0.001$ ), and allergy to sunflower seeds and mixed grasses $(r=2.0, P<0.0001)$.

As regards to allergy to insects, there was also positive correlation between allergy to common wasp venom and honeybee venom ( $r=0.03, P=0.001)$, and common wasp venom and cockroaches $(r=0.3, \quad P=0.01)$. Such correlations could be explained by the presence of protease allergens in the extracts obtained from the insects which probably the cause of allergy as previously mentioned. As regards to food allergy there was a positive correlation between allergy to milk and egg white ( $r=0.46$, $P<0.0001)$, and between Solaneceae and tomato $(r=0.46, P<0.0001)$. These associations deed further studies to confirm and explain them.

In conclusion, our study provided useful information on the pattern of allergen sensitization in this part of the country. Although, avoidance of the allergens may be difficult in respect to aeroallergens, while contact and food allergens can be avoided more easily. Therefore, it is advisable for the patient to do SPT to identify the allergen to which he/she is sensitized. 


\section{Author Contributions}

ANT is responsible for ensuring that the descriptions are accurate and agreed by all authors; ANT, manuscript idea; ANT and KSA, practical work; KSA, responsible for the clinical evaluation and diagnosis of bronchial asthma; ANT, final supervision.

\section{Declaration of Conflicting Interests}

The author(s) declared no potential conflicts of interest with respect to the research, authorship, and/or publication of this article.

\section{Funding}

The author(s) denies receipt of any financial support for the research, authorship, and/or publication of this article.

\section{Ethical approval}

The study protocol was reviewed and approved by the local ethical committee of the Faculty of Medicine, Sohag University (dated February 2019).

\section{Informed consent}

Patients signed informed consent, indicated that they agreed to have the skin prick test. For children below 18 years the consent was signed by their firstdegree relatives. Willingness of children to take the skin test was considered their assent.

\section{References}

1. Tanno LK, Calderon MA, Smith HE, et al; (2016). Joint Allergy Academies. Dissemination of definitions and concepts of allergic and hypersensitivity conditions. World Allergy Organ J.; 9:24.

2. Braunstahl GJ. (2009). United airways concept: what does it teach us about systemic inflammation in airways disease? Proc Am Thorac Soc. 6:652-4.

3. Pawankar R. (2012). The increasing complexity and burden of allergic disease and asthma in the asia pacific region. World Allergy Organ J. 1-2.

4. Pawankar R. Canonica G, Holgate S, Lockey R. (2012). WAO, white book on allergy. Milwaukee (WI): World Allergy Organization. 27-38.

5. Brozek JL, Bousquet J, Baena-Cagnani CE. (2010). Allergic rinitis and its impact on asthma (ARIA) guidelines. J Allergy Clin Immunol. 126: 466-76.

6. Pawankar $\mathrm{R}$, Baena-Cagnani $\mathrm{CE}$, Bousquet $\mathrm{J}$. (2008). State of world allergy report 2008: allergy and chronic respiratory diseases. World Allergy Organ J. 1:S4-17.
7. Schneider L, Hanifin J, Boguniewicz M, et al; (2016). Study of the atopic march: development of atopic comorbidities. Pediatr Dermatol. 33:388-98.

8. van der H, Annelies E, Klip H. Brand PLP. (2007). Risk of developing asthma in young children with atopic eczema: a systematic review. J Allergy Clin Immunol. 120:565-9.

9. De Jong AB, Dikkeschei LD, Brand PL. (2011). Sensitization patterns to food and inhalant allergens in childhood: a comparison of non-sensitized, monosensitized, and polysensitized children. Pediatr Allergy Immunol. 22: 166-171.

10. Global Initiative for Asthma. Global strategy for asthma management and prevention (2016 update). Global Initiative for Asthma; 2016.

11. Mokdad $\mathrm{AH}$, Forouzanfar $\mathrm{MH}$, Daoud $\mathrm{F}$, et al; (2016). Global burden of diseases, injuries, and risk factors for young people's health during 1990-2013: a systematic analysis for the Global Burden of Disease Study 2013. Lancet 387: 2383-401.

12. Nurmagambetov $T$, Khavjou $O$, Murphy L, et al; (2017). State-level medical and absenteeism cost of asthma in the United States. J Asthma 54: 357-70.

13. Selner JC, Sullivan TJ, Ahlstedt. (2001). Current issue relating to in vitro testing for allergen-specific IgE: a work-shop report. Ann Allergy Asthma Immunol. 82: 407-412.

14. Pawankar R, Canonica GW, Holgate ST, et al; (2013). The world allergy organization (WAO) white book on allergy. Update 2013. WAO:87-92.

15. Australasian Society of Clinical Immunology and Allergy. Skin prick testing for the diagnosis of allergic disease. Balgowlah (Australia): Australasian Society of Clinical Immunology and Allergy Inc. (2013); 1-39.

16. Van Cauwenberge $P$, Bachert $C$, Passalacqua $G$, et al., (2000). Consensus statement on the treatment of allergic rhinitis. European Academy of Allergology and Clinical Immunology. Allergy; 55: 116-34.

17. Ferastraoaru D, Shtessel M, Lobell E, et al; (2017). Diagnosing environmental allergies: Comparison of skin-prick, intradermal, and serum specific immunoglobulin E testing. Allergy Rhinol. 8:53-62.

18. O'Hollaren MT, Yunginger JW, Offord KP, et al; (1991). Exposure to an aeroallergen as a possible precipitating factor in respiratory arrest in young patients with asthma. N Engl J Med.; 324:359-63.

19. Chinn S, Jarvis D, Luczynska C, Burney P. (1998). Individual allergens as risk factors for bronchial responsiveness in young adults. Thorax. 53:662-67.

20. Berger A. Skin prick testing. $\mathrm{Br}$ Med J. 2002; 325:414.

21. Bharti Chogtu, NehaMagaji, Rahul Magazine, et al; (2017). Pattern of Allergen Sensitivity among Patients with Bronchial Asthma and/or Allergic 
Rhinosinusitis in a Tertiary Care Centre of southern India. Journal of Clinical and Diagnostic Research. 11(8).

22. Patel A, Choudhary S. (2012). Prevalence of allergen sensitivity in nasobronchial allergy in Gujarat, India. Natl J Med Res. 2(4):431-34.

23. Prasad R, Verma SK, Dua R, et al; (2009). A study of skin sensitivity to various allergens by skin prick test in patients of nasobronchial allergy. Lung India. 26:70-73.

2. Kumar R, Sharan N, Kumar M, et al; (2012). Pattern of skin sensitivity to various aeroallergens in patients of bronchial asthma and/or allergic rhinitis in India. Indian J Allergy Asthma Immunol. 26:66-72.

25. Agrawal RL, Chandra A, Jain S, et al; (2008). Identification of common allergens by SPT associated with united airway disease in Allahabad, Uttar Pradesh, India. Indian J Allergy Asthma Immunol. 22(1):7-13.
26. Patel A, Choudhary S. (2012). Prevalence of allergen sensitivity in nasobronchial allergy in Gujarat, India. Natl J Med Res. 2(4):431-34.

27. Sarinho E, Mariano J, Sarinho S, et al; (2009). Sensitisation to aeroallergens among asthmatic and non-asthmatic adolescents living in a poor region in the Northeast of Brazil. Allergol Immunopathol (Madr); 37(5):239-43.

28. Arabkhazaeli A, Vijverberg S, van Erp F, et al; (2015). Characteristics and severity of asthma in children with and without atopic conditions: a crosssectional study. BMC Pediatr. 15:172.

29. Liu A, Jaramillo R, Sicherer S, et al; (2010). National prevalence and risk factors for food allergy and relationship to asthma: results from the National Health and Nutrition Examination Survey 2005-2006. J Allergy Clin Immunol. 126 (4):798-806. 